\title{
Lapurdum
}

Euskal ikerketen aldizkaria | Revue d'études basques |

Revista de estudios vascos | Basque studies review

$14 \mid 2010$

Numéro XIV

\section{Le paratexte débâillonné (I) : retour sur la Muse bayonnaise,ca 1620, texte occitan perdu}

\section{Chistian Bonnet}

\section{OpenEdition \\ Journals}

Édition électronique

URL : http://journals.openedition.org/lapurdum/2262

DOI : 10.4000/lapurdum.2262

ISSN : 1965-0655

\section{Éditeur}

IKER

Édition imprimée

Date de publication : 1 octobre 2010

Pagination : 7-32

ISSN : 1273-3830

Référence électronique

Chistian Bonnet, «Le paratexte débâillonné (I) : retour sur la Muse bayonnaise,ca 1620, texte occitan perdu », Lapurdum [En ligne], 14 | 2010, mis en ligne le 15 septembre 2014, consulté le 20 avril 2019 URL : http://journals.openedition.org/lapurdum/2262 ; DOI : 10.4000/lapurdum.2262 


\title{
Le paratexte débâillonné (I): retour sur la Muse bayonnaise, ca 1620, texte occitan perdu
}

\author{
Chistian BONNET \\ Université Blaise Pascal, Clermont-Ferrand
}

En hommage à Robert Darrigrand, éveilleur de langues, d'études et d'âmes, avec gratitude et amitié.

Si la littérature d'oc médiévale jouit d'une notoriété universelle grâce à l'aura des troubadours et du trobar, au point que de nombreux manuels de français en annexent la matière qui ignorent superbement la suite de la production d'expression occitane, la littérature moderne et contemporaine pâtit fortement du monolinguisme que la France promeut dans l'espace hexagonal. Aussi les études occitanes sont-elles pour ces périodes très en retard sur le niveau de connaissance des autres littératures européennes soit de langues d'Etats, comme le néerlandais par ex., soit de langues sans Etat, comme le catalan. Malgré une récente et timide libéralisation sur ce plan, de nombreux pans de la littérature d'oc moderne restent donc mal connus privant le public, en premier lieu occitan lui-même, de l'accès à ce patrimoine (la France ne forme que quatre professeurs d'occitan par an pour cette langue couvrant le tiers de son territoire). Les progrès se sont concentrés sur l'édition d'œuvres selon les critères textologiques contemporains, mais l'exégèse de cette production reste fort lacunaire, aussi bien dans ses prolongements historico-culturels et sociolinguistiques que pour l'ensemble des études spécifiquement littéraires. Nous proposons ici son approche par l'examen d'éléments par nature révélateurs pour une littérature minorée que sont les paratextes, éléments textuels et éditoriaux produits en vue ou à l'occasion de la publication des œuvres.

Le paratexte de la littérature occitane n'a guère fait l'objet d'études spécifiques (1). La récente et heureuse réédition par Xarles Videgain d'un guide de conversation commerciale trilingue du début du XVII ${ }^{\mathrm{eme}}$ s. (2), nous offre l'opportunité d'examiner de plus près (3) un tel document paratextuel, plus précisément un péritexte puisque celui-ci était par destination voué à être joint à l'ouvrage (4). En effet, l'exemplaire ainsi reproduit de l'Interprect, œuvre de Voltoire dont le nom, sinon la biographie, est bien connu des parémiologues basques et occitans (5), présente in fine (après le mot FIN, page 280 et dernière) une table des matières en apparente concurrence à celle placée en tête du volume. Le lecteur n'est pas long à s'apercevoir (6) que seule la table initiale annonce pertinemment le contenu et la pagination du guide, en 
conformité avec le titre et le sujet de l'ouvrage, alors que la table placée à la fin renvoie à une matière de nature différente et à un texte de pagination exogène au corps du volume. C'est de cette table d'un ouvrage absent, péritexte attestant de textes occitans gascons inconnus, que nous voudrions ici tirer quelques renseignements.

Afin de mettre le lecteur à même de suivre le décryptage que nous en proposons, il est préférable de reproduire tout d'abord avec exactitude cet ultime feuillet de table signé * , de même format oblong et imprimé recto-verso selon la même disposition graphique que la table initiale, avec un matériel appartenant manifestement au même atelier.

\section{Recto :}

Centré : [ bandeau : rinceau avec oiseaux ] /

TABLE DV CONTENV EN CE LIVRE /

Justifié : [ à gauche, sur toute la hauteur des sept lignes du texte en italiques, lettre ornée droite 'P' ]

REMIERE partie contenant le subiect $E$ dif- / pofition de louure aulx Amys \& Compatriots / à folio i. au premier liure intitulé RETIRADE. / LA Mufe Baionnoife en ladicte premiere partie / à fol. 20. I

LA Conuerfation Ciuile pour le Temps prefent / audict liure à folio 33. I

Signature :

[ même étoile à cinq branches qu'en première page de la table initiale, à mêmes hauteur (ligne suivant le texte) et retrait à droite (d'un tiers) que celle-ci et que dans le corps de l'ouvrage. ]

Verso :

Justifié : $\quad$ L A Nourriture des enfans aulx Peres E Meres en vers Gafcons audict / liure à folio 60.

Lexhortation E remedes aulx defbauchés E difolus. à fol. 76. /

Centré :

STANCES EN VERS GASCONS /

Justifié : $\quad$ Pour E Contre les Blaphemateurs. En la dite premiere partie du prefent / liure à folio 96.

Contre les Yurognes, Gourmands, E Prodigues. prem. part. à fol. 105 /

Contre les Pipeurs E Mauuais Ioueurs . prem. part. à folio 113.

[ Nota que cette dernière ligne de texte se situe très au-dessus de la marge inférieure de la mise en page, à la hauteur de la ligne 'LA Muse Baionnoife...' du recto. ]

Nous tenterons d'abord, par un questionnement matériel adéquat, de " faire parler » des textes auxquels elles réfèrent ces quelques lignes rédigées à leur périphérie (d'où le soin pris dans la note 3 et la reproduction ci-dessus pour le respect des accents, orthographe ou cacographies: interpre(s)t, bla(s)phémateurs, ponctuation, typographie, mise en page), afin de 
recueillir toute l'information qu'elles recèlent sur les plans successivement éditorial et textuel, puis sociolinguistique et d'écriture, avant d'examiner si ce paratexte conforte nos connaissances sur l'institution littéraire occitane.

Nous commencerons par l'aspect éditorial, à propos duquel deux faits retiennent l'attention. Le premier est le réalisme descriptif de la seconde table et sa pagination positive de l'ouvrage afférent «si minutieuse qu'aucun doute sur son existence n'est possible », selon le mot de Gustave Brunet, qu'il parâit difficile de contredire, sauf à préciser l'état d'avancement de cette impression. Le deuxième fait est naturellement l'adjonction de feuillet supplémentaire exogène, intrinsèquement non daté, à un ouvrage manifestement déjà complet et achevé puisque conforme à la table de matières à lui dévolue. Celle qui suit le titre est en effet seule explicitement dédiée: 'TABLE DE / LINTERPREST [sic] OV...', insérée comme on vient de voir entre les premier et second feuillets du premier cahier. Annoncée au titre comme 'dernier escripte', que nous interprétons comme rédigée en dernière instance c'est à dire après la composition typographique, ainsi que le confirme son insertion matérielle, sa postériorité en garantit l'exhaustivité descriptive (faisant revue des diverses parties composant l'ouvrage 'ainsi qu'est contenu en la table') autant que l'exactitude de pagination, l'une et l'autre désormais aisément vérifiables grâce à l'initiative de X. Videgain. Incontestablement erronée, cette adjonction en fin de volume de la table d'un autre ouvrage peut cependant être diversement analysée.

S'agit-il d'une erreur 'intellectuelle' ayant conduit le personnel de l'atelier à confondre les constituants (corps des volumes et tables respectifs) de deux ouvrages simultanément sous presse, ce qui pourrait signifier une communauté de traitement de ces éditions? Celleci pourrait résulter d'une provenance commune des manuscrits : cette lointaine impression basque à Lyon, même s'il s'agit d'une place commerciale active, devient moins surprenante chez le typographe Rouyer dont l'atelier a longtemps été actif dans le sud de la Gascogne, où il avait pu conserver des relations (7) ; mais cette caractéristique foraine commune présentait un risque d'assimilation des deux ouvrages pour un personnel peu préparé à distinguer le basque du gascon. Par extension on peut leur supposer une commande identique, par exemple d'un libraire bayonnais désireux de constituer un fonds quelque peu diversifié par les thèmes et surtout les publics, le gascon venant ici s'ajouter aux trois langues de l'Interprect. On n'oubliera pas enfin, quand même rien n'assure que Voltoire ait quelque point commun avec le nouvel ouvrage, l'éventualité d'un même auteur, puisqu'on sait par ailleurs notre interprète s'être particulièrement intéressé aux proverbes gascons, dont il a effectué une présentation versifiée dans cette langue, qu'il faut par conséquent lui supposer maternelle (8). De tels cas de figures ont pu induire une sorte 'd'extension conceptuelle' entre les deux éditions d'autant plus compréhensible qu'il y a lieu de croire ces tables (surtout celle de l'Interprect dont se vérifie l'exactitude de pagination) avoir été rédigées dans ou par l'atelier. Il serait alors concevable de rencontrer d'autres exemplaires semblablement 'augmentés'.

Ces trois hypothèses ouvrent des perspectives également attractives, mais ne permettent cependant pas d'exclure la banale erreur matérielle, de manipulation : le feuillet qui traînait là, tirage d'essai ou rebut d'un ouvrage déjà ancien (toutefois de même format), saisi par inadvertance lors de l'assemblage d'un nouvel ouvrage ; mais en ce cas saisi lors de la confection 
singulière d'un exemplaire déterminé. Car l'exemplaire de Bordeaux constitue pour l'heure un unica, rendant hasardeuse toute spéculation d'intentionnalité ou du moins de signification éditoriale dans cette adjonction. Or l'on vient d'observer, ce que nous conforterons plus loin, que le verso de ce feuillet final, la table de la Muse, présente tout l'aspect d'une composition inachevée. L'ouvrage entrepris lui-même fut-il jamais achevé et, la composition commencée, la copie en était-elle parvenue dans sa totalité à l'atelier ? Labsence de date d'impression au titre de L'Interprect doit-elle être mise en rapport avec une incertitude de cet ordre?

Lemplacement laissé vierge en dernière ligne, au bout de l'adresse (voir note 3), pourrait être un indice en ce sens aussi bien qu'un argument de vente visant à palier le vieillissement de l'ouvrage. On doit certes créditer d'un solide esprit commercial un imprimeur comme Rouyer ; il n'eût pas manqué de chercher à écouler un ouvrage imprimé, pour incomplet qu'il soit, dès lors que constitué, comme ici le recueil de la Muse, de pièces mêlées dont le choix peut être librement arrêté. Mais force est de constater pour l'heure l'absence de produit bibliographique connu correspondant en propre à la table superflue. La partie très certainement composée, futelle réellement imprimée et, supposée imprimée, ne put-elle avoir été victime d'un accident matériel, notamment à la suite d'une interruption du tirage ? Faute d'éléments probants, il nous faut donc laisser en suspens ces considérations éditoriales.

Essayons maintenant d'entreprendre l'exégèse de ces quelques lignes en leur qualité de table des matières, reflétant la teneur d'un corpus textuel hors d'atteinte. Nous proposons d'en cerner la valeur signalétique dans le sémantisme lexical, concurremment aux dispositions graphiques. La table finale est ainsi dite 'du contenu en ce livre', ce qui pose la question du sens du mot livre selon qu'on entend désigner un volume, aujourd'hui registre relié, ou le texte, œuvre ou subdivision d'œuvre écrite, susceptible de contenir dans un tel volume. Pour éclairer ce point, observons l'emploi presque consécutif des mots partie, ouvre et livre dans les lignes contiguës. Le 'contenu' s'ouvre en effet par une 'première partie' indiquant la 'disposition de l'ouvre' au folio 1 du 'premier livre'. La première partie réfère par conséquent, en l'absence d'indication restrictive, à l'œuvre entier, appréhendé en ses premiers mots dès le folio un (que nous proposons de comprendre page une, à la suite de l'emploi constant du mot folio comme synonyme de page puisqu'aussi bien l'Interprect est effectivement paginé). Par répétition de la préposition à, ces premiers mots sont derechef localisés 'au premier livre', lequel se voit identifié en place de pagination par un 'intitulé' propre. La cohérence de cette terminologie, parallèle à la pagination fournie, au sein de la même phrase nécessite donc ici l'acception du mot livre comme 'subdivision d'un ensemble textuel', ou pour mieux dire d'un hypertexte, constituant la première partie d'un ensemble suffisamment complexe pour recevoir l'appellation superlative d'œuvre, dont il convient en effet d'expliciter la disposition interne au lecteur.

Nous comprenons ainsi que le terme en capitales de RETIRADE, constitue l'intitulé du premier livre et, conséquemment, que 'LA' Muse baionnoise... puis 'LA' Conversation Ciuile... constituent les intitulés respectifs des second et troisième livres appelés par le premier emploi d'un adjectif ordinal, non qualifiés comme tels, mais explicitement inclus, s'agissant de la Muse, 'en ladicte première partie'. En effet, rattachée à la première partie, la Muse est logiquement incluse au rang de ses subdivisions (hypotexte) et corrélativement soustraite au premier livre qui venait précisément d'être lui-même présenté comme hypotexte de cette partie. On peut en outre observer, à l'intérieur de l'alinéa plutôt homogène constitué par ces trois phrases à droite de la lettre ornée, le souci du typographe de faire ressortir le mot initial des deux 
dernières phrases : il y procède à la fois visuellement, par un recours délibéré aux majuscules aligné sur l'emploi de celles-ci dans le mot PREMIERE (où il était de convention à la suite de l'ornement), et syntaxiquement par le recours à un article présentatif, semblablement aligné sur le précédent emploi du contracté défini au (soit : à LE livre, pour celui intitulé Retirade).

Cet emploi du mot livre parfaitement approprié à l'usage littéraire (il est ici affaire de Muse et d'éloquence) contraste rétroactivement avec la première occurrence du mot (ligne 1 : 'Table du contenu en ce livre') qui doit pour sa part être comprise comme renvoyant à la matérialité physique du volume sorti des presses, ne préjugeant aucunement de la disparité des contenus textuels dont la table reçoit fonction, justement, de déployer la cartographie. Il serait d'ailleurs tout à fait singulier que l'intitulé de ce volume ne se trouve dévoilé que dans sa table des matières. Quand bien même la publication projetée eût placé la table en tête, l'intitulé Retirade' fût resté à la trappe dans un volume sans titre! A défaut d'une rigueur terminologique dont l'époque n'était pas encore prodigue - mais l'ambivalence du mot livre subsiste aujourd'hui - il faut convenir que les deux acceptions successives, pour différentes qu'elles soient, entrent en cohérence sémantique : 'dans ce volume le lecteur trouvera une œuvre réunissant plusieurs parties'.

Il semblerait pourtant que cette interprétation puisse être démentie par la dernière phrase du recto, puisque la Conversation Ciuile y est localisée 'à folio 33 audict livre', renvoi au sus-dit qui ne peut viser la phrase précédente (où la Muse est localisée, comme on a vu, par appartenance à la partie) et paraît en conséquence référer au premier item : au livre intitulé Retirade. Cette objection ne nous paraît pas consistante car nous pensons que le rédacteur, poursuivant son parallèle entre matérialité éditoriale et délimitation textuelle, réfère ici à la mise en page du titre de départ de la Conversation dans la visibilité conférée par l'usage typographique contemporain, rendue extrêmement opérante grâce au bandeau orné placé en surtitre et au grand corps de caractères requis pour l'intitulé d'un nouveau livre. Le rédacteur se conforme ainsi à sa formulation précédente, doublant l'indexation numérique de la pagination par le renvoi à un repérage visuel global localisant les sections 'au feuilleter' grâce à la surcharge graphique de leurs pages de titre et, accessoirement, à leur indexation structurelle.

Ces constatations vont en effet se renouveler pour les deux items figurant en tête du verso : LA Nourriture... aux Pères et Mères localisée dans l'ordre inverse 'audict livre à folio 60' puis Lexhortation... aulx desbauchés pour lequel l'indication du livre d'appartenance a été désormais spontanément ou délibérément jugée superfétatoire. Avec ce cinquième livre s'interrompt une série en apparence hétérogène par l'annonce de Stances en vers gascons dont l'intitulé augure une homogénéité simultanément générique et linguistique. Toutefois, comme on a pu le constater, cette ligne typographique à la différence des précédentes est d'une part dépourvue de localisation (soit pagination, soit structurelle) d'autre part centrée et composée en capitales ; le soin porté à la seconde caractéristique rendant improbable l'origine fortuite de la première. Autrement dit, il s'agit du titre d'une subdivision notoire du corpus textuel, comme si la table voulait restituer la présence d'une page de titre de départ. L'emploi de capitales le place au niveau de RETIRADE, mais sans renvoyer à un fragment individualisé, localisable en tant que tel par la pagination ou une assignation structurelle de sixième livre, qui reste implicite. Ce sont les subdivisions énumérées à la suite qui vont fournir ces références en même temps qu'un intitulé de leurs sujets. Car le terme de stances, s'il suffit à annoncer un lot de poèmes de cette forme générique, reste muet sur leur thématique, et ce silence vient ici en rupture avec l'exposé, plutôt disert, de l'objet de chacune des pièces précédemment énumérées. 
Aussi les trois items suivants apparaissent-ils comme la teneur des stances promises : sinon un ensemble homogène, du fait de paginations propres, du moins une succession logique. C'est ce qu'induit, à en juger par les deux premiers, un nombre de pages sensiblement plus réduit pour ces poèmes à développement en effet ordinairement limité (l'incertitude prévaut pour le troisième, à le supposer final, en l'absence de pagination consécutive sur le reste du feuillet). C'est aussi ce que soulignent leurs sujets : blasphème, ivrognerie, gourmandise et prodigalité, tricherie et jeu, soit autant de péchés contre la Religion et la morale. Notre Muse persévère dans une voie résolument didactique : civilité de la conversation, exhortation contre la débauche et maintenant stances contre les vices ; 'l'œuvre' annoncé, pour pratiquer une esthétique du discontinu (pièces, sujets, formes, et langues variés), ne manque pas de cohérence intellectuelle. La combinaison du graphisme et de la syntaxe conforte ainsi le sémantisme. Ceci permet d'éclairer l'inclusion structurelle 'en la première partie' assignée à ces trois stances. L'importante césure graphique créée par le titre de ce livre de stances (par le corps, le centrage et les entrelignes) s'il explicite la subordination solidaire des trois stances listées, ne rompt pas l'unité de la première partie ainsi continuée.

Une difficulté subsiste cependant pour la première stance, sur les blasphémateurs, localisée 'en la première partie du présent livre' selon une hiérarchie en apparence inverse de celle que nous venons de dégager. Aussi doutons-nous de l'incohérence que tend à introduire une première lecture soudée des mots 'partie du livre' (perçus comme formant un syntagme soudé 'nom + complément'). En effet, les livres rencontrés jusqu'ici ont été exclusivement référencés soit à la première partie, soit à eux-mêmes, selon une alternance certes aléatoire mais n'excluant pas l'autre formule, de fait synonyme. Par contre nous n'avons relevé aucun cas de combinaison de ces localisations : 'à la partie de tel livre' ou 'au livre de telle partie' établissant une arborescence. Aussi pensons-nous avoir affaire à une simple co-occurrence des deux formules d'indexation. Celle-ci est masquée par l'absence ordinaire de ponctuation (la virgule n'est employée qu'en énumération et dédaignée en syntaxe) à l'occasion d'une coquille typographique assez banale dans la composition du mot $d u$ que nous proposons de lire $a \boldsymbol{u}$ : 'en la première partie, au présent livre, à (la page) 96', suivant le procédé utilisé pour la Conversation ciuile.

Reste que cette première partie, riche de six livres, dont un de stances, demeure veuve de la seconde, logiquement appelée par l'emploi de l'adjectif ordinal de départ. Comment expliquer alors que la page soit demeurée blanche dans son tiers inférieur, vierge de la suite de l'œuvre? Un ou plusieurs livres supplémentaires reçus ou escomptés par l'atelier étaient-ils en attente d'inscription? Une autre explication est suggérée par l'insuffisance de place restante pour permettre l'emploi d'un ornement typographique de corps important, sur le modèle de mise en page adopté en première partie, avec une nouvelle lettre ornée destinée à indiquer la partie suivante. En effet le code typographique alors en usage répugne à laisser une page incomplète sans indication de l'achèvement du texte, au moyen du mot FIN comme on l'a déjà vu, ou d'un ornement typographique de taille proportionnée au volume du blanc, voire l'emploi des deux comme on le voit aussi, précisément, en page finale de la table initiale.

Cette situation ambiguë conduit à se demander si la disposition que nous constatons ne pourrait pas résulter d'une hésitation sur l'agencement de l'éventuelle seconde partie. On sait que le terme de 'partie' est d'un emploi fréquent dans les volumes d'œuvres mêlées de l'époque moderne, recevant parfois dans le domaine poétique des noms imagés de la collecte ('bouquets' ou 'Ramelets') ou de l'ordonnancement (avec métaphores temporelles 'journées' et 'serées', 
ou spatiales 'parterres' et 'quoarrèus'). Les presque 130 pages imputables à la part décrite de l'oeuvre paraissent, malgré la limite du format, suffire à la substance d'une partie de taille convenable. A-t-il été envisagé, comme on en voit divers exemples que cette partie formât le tout de la publication, constituant à elle seule le volume, la suivante devant donner lieu à un volume distinct? La littérature occitane offre suffisamment d'œuvres perdues, et même plus récemment retrouvées, voire de taille conséquente et dans la même veine, pour en autoriser l'hypothèse (9). A l'inverse s'agirait-il d'un indice, comme évoqué ci-avant, d'inachèvement ou d'interruption absolus du processus éditorial ? Nous ne pouvons alors déterminer si cette dernière concerne l'édition seulement (abandon de la publication et donc de la composition) ou l'opus à éditer (par défaillance de sa rédaction, ou par indisponibilité de sa copie).

La table finale conserverait ainsi trace de la seule velléité d'un ouvrage plus ample, qui eut compté deux parties ou plus, dans lequel l'occitan semblait promis à une place non négligeable. Consolons-nous en nous persuadant, en revanche, de la réalité de la (première) partie dont l'énumération détaillée (intitulés) du contenu imprimé (paginé) ne laisse aucun doute sur l'existence des textes correspondants. C'est sur ceux-ci qu'il nous faut revenir pour en mieux observer l'apport sociolinguistique et littéraire.

Le premier livre est donc intitulé 'Retirade', mot qui ne paraît assimilable à aucune forme linguistique française, notamment du fait de l'emploi du suffixe flexionnel-ade, paradigme des passés composés féminins de l'occitan, ici réalisé avec un -e final post-tonique caractéristique des parlers de la façade maritime de la Gascogne (Maremne). Exact équivalent néo-roman du français retraite, Retirade est ainsi évocateur des poétiques humanistes de l'introspection (10). Néanmoins l'emploi de cette forme gasconne authentique paraît insuffisant pour inférer formellement l'emploi de l'occitan dans ce premier livre, dès lors que le recours à cette langue, à l'instar de la pagination, est ordinairement spécifié en sus de l'intitulé du fragment concerné. A vrai dire, Retirade ne l'interdit pas davantage et si l'objet de ce livre est, comme indiqué, de présenter au lecteur 'le sujet et disposition de l'ouvre', la présence même partielle de l'occitan n'est pas à exclure de cette introduction. Ne peut non plus être écarté a priori l'éventuel emploi d'un langage mixte francitan (11) mais l'usage de celui-ci, habituellement réservé aux registres satirique ou burlesque, paraît ici hors de sujet.

En tout cas cette partie, explicitement adressée 'aulx amys' lecteurs et très probablement écrite en prose comme tend à le prouver la mention explicite de vers pour d'autres pièces, semble très exactement correspondre à une préface d'auteur, ou pour l'exprimer dans les concepts de l'époque, à la phase rhétorique de la 'captatio benevolentiae' initiale. Elle révèle donc un auteur au fait de l'art de la persuasion sinon de l'éloquence, mais aussi suppose un ouvrage d'une certaine exigence pour le lecteur, requérant en conséquence la clarté d'exposition comme condition à son adhésion au pacte de lecture. L'emploi du français dans ce livre, à l'instar de la table qui le recense, peut constituer un élément de séduction dans ce sens. Mais il est en vérité moins destiné au pouvoir linguistique francophone (institutions royales, encore lointaines pour la frontalière Bayonne, et noblesse seconde, également absente de la ville) habituellement flatté, qu'aux 'amis' par le commerce, au nombre desquels la clientèle étrangère, dont ces nombreux Flamands pratiquant le français, et ces Espagnols occupants des précédants.

Le second livre conserve également une part d'indécision s'agissant aussi bien de l'emploi 
de l'occitan gascon potentiellement suggéré par l'ethnique baionnoise' (supposé que nous restions exempts de la contamination induite par l'activité polyglotte de Voltoire, que rien n'assure pourtant être l'auteur), que du choix du registre d'écriture qu'on incline, pour une muse, à croire plus convenable en vers. Rappelons d'ailleurs, là aussi, la possibilité très réelle d'un texte mixte, que ce soit par une mise en dialogue des langues en présence, nous trouvant, en Gascogne, à l'épicentre du modèle trilingue de G. Salluste Du Bartas par strophes alternées (12), ou par dilection pour le discontinu, mêlant prose et vers dans le sillage de l'Astrée, voire par combinaison des deux. Ce qui est sûr est que le naturel bayonnais de cette muse la situe dans le prolongement des dédicataires du livre premier : amys mais aussi compatriots de l'auteur, pour la plupart sans doute simultanément. Drainant le commerce gascon de la vallée de l'Adour, et au-delà jusqu'à Auch et Toulouse, la cité maritime rivale de Bordeaux est donc, avec ses singularités, à l'origine de son inspiration (13).

Avec son intitulé rappelant irrésistiblement la Civilité morale des enfants d'Erasme et autres guides de conversation, le tiers livre nous place à un carrefour générique et culturel. D’une part, le syntagme 'conversation civile' renvoie à un entretien moral, de tonalité effectivement assortie à la retirade, et certainement disposé en forme dialoguée. Les mots propos, entretien, dialogue, conversation, désignaient à l'époque moderne, de façon à peu près synonyme, des exposés polémiques ou didactiques à progression déductive par recours à des interlocuteurs de convention. Ces textes dialogués, répandus aussi dans le domaine occitan, constituaient autant de versions profanes et littérarisées du catéchisme par questions et réponses, de tonalité tantôt grave, tantôt burlesque (parodiques) puis ironique (sous les Lumières). Ladjectif civil renvoie à l'organisation sociale dans son ensemble, installant la société à la fois comme l'objet de cette conversation et comme sa qualité. Celle-ci, la civilité, est, de façon générale le produit d'une éducation soignée, marque de distinction qui revient en faveur après les troubles de religion, remplaçant la tragédie par la pastorale, avec d'Urfé, Larade ou Goudouli, l'affrontement par la galanterie autour de Madame de Rambouillet ou dans les salons toulousains. Une telle conversation s'éloigne donc de la férule des pédants du siècle précédent pour en appeler à l'honnête homme, civil et vésiat (14).

Est ainsi proposée comme appropriée au 'temps présent' une conversation d'ordre général et de caractère sociable entre citoyens, dialogue en conséquence pacifique, conduit en termes civils, de protagonistes naguère pourtant déchirés par une guerre non moins dite civile, entendez générale, de tous contre tous, fratricide entre chrétiens, funeste à la société. Naguère déchirés? Globalement, c'est évidemment à la période des conflits armés confessionnels que la locution temps présents s'oppose allusivement, aujourd'hui supplantant hier. La référence est d'autant plus prégnante pour les Aquitains, et spécialement les Béarnais, que leur Roi, acteur majeur de ces conflits, est ensuite devenu le promoteur de la paix et Roi de France par surcroît, régnant, lui aussi, sur deux langues. Mais la chronologie pourrait être affinée. S’il eut été vain, dans nos hypothèses éditoriales, de supputer le titre de l'ouvrage perdu, la sorte de communauté de destin que nous croyons s'être un moment établie entre l'Interprect et son jumeau perdu les rapproche en tout cas de façon manifeste par la date. Si rien n'autorise à attribuer de façon certaine le millésime manuscrit non vérifié de 1620 à la date de parution putative de l'œuvre (et encore moins de ses parties), tout nous engage à les estimer proches.

C'est alors pour le Béarn et ses abords le début d'une période de tensions extrêmes liées au sort du Royaume. Lorsqu'Henri III de Navarre avait réuni deux sceptres en ses mains (régime d'union personnelle), le statut de l'Etat béarnais n'avait guère été modifié. Sans doute les 
'papistes' minoritaires espéraient-ils beaucoup du rapprochement avec la France, où l'abjuration du nouveau roi consacrait la prééminence catholique. Cependant le Parlement de Navarre ne pouvait renier les constitutions autochtones, ni la minorité catholique réclamer uniment l'abolition de la souveraineté. Un parti national et protestant, d'inspiration monarchomaque, discret du vivant d'Henri, s'est fait de plus en plus virulent (15) au fur et à mesure que la régente de France et son fils, catholiques zélés d'orientation absolutiste, sont intervenus politiquement pour établir le catholicisme comme religion d'état dans le pays ('cujus regio, ejus religio'). Les libelles des deux bords, dans lesquels l'occitan trouve place en prose et en vers, témoignent de l'acuité du conflit (16). Louis XIII fut dans l'obligation de venir à Pau, le 20 octobre 1620, usant de sa qualité de suzerain, mais non sans appui militaire, pour faire enregistrer un Edit d'Union, mettant fin de facto à l'autodétermination de la Navarre, et ouvrant un conflit général avec l'ensemble du protestantisme occitan, de La Rochelle aux Cévennes. La résistance béarnaise continua néanmoins plus de deux ans, conduite par Jean-Paul de Lescun, finalement saisi et exécuté à Bordeaux le 19 mai 1622.

On comprend sans peine l'intérêt qui pouvait donc s'attacher, en 1620 ou immédiatement avant, pour un ressortissant de la cité maritime, république commerçante encore très imparfaitement assujettie au régime français, et placée entre l'écorce et l'arbre de ces deux voisins, à engager entre 'compatriots', que nous comprenons ne point désigner les régnicoles français, des conversations en effet civiles, aux deux sens du terme, pour exorciser les dangers 'du temps présent'. Intérêt aiguisé par le siège d'un évêché dont relèvent jusqu'en 1566 quatre archiprêtrés basques de l'ancienne Navarre cis-pyrénéenne (toujours revendiqués, pour l'heure occupé par Jacques de Mauri, prélat énergiquement engagé dans la contre-Réforme. Or celuici heurte, par ses manières fortes, un conseil de ville par nature hostile au scandale comme à la sédition, qui entend conserver par rapport aux Réformés tant urbains que forains (Anglais, Flamands et Nordiques liés au commerce) une attitude sinon laïque, du moins irénique, où la modestie se fait instrument de modération afin de « vivre en paix et repos, n'injurier ni les uns ni les autres, ni se reprocher les uns les autres leurs qualitez » (J. Pontet, op. cit. en note 13, p. 168).

Plus difficile est de déterminer si la conversation de ce tiers livre, qui paraît en effet exempte du caractère polémique des pièces liées au conflit béarnais, fait un semblable emploi de l'occitan, ni de la versification, dont l'absence de mention explicite laisse présumer le double refus. Il en va tout à l'inverse du livre quatrième, dont le titre spécifie immédiatement la combinaison 'en vers gascons'. La tonalité n'en paraît pas moins morale sous le titre LA Nourriture des enfans aulx Peres et Meres. Mais il ne s'agit pas d'une version puérile de la conversation précédente, car la phrase doit être entendue à rebours de la syntaxe actuelle. Des n'y a pas le sens attributif ('nourriture pour enfants') mais distributif ('dispensée par eux'). Et l'on comprend ainsi que l'aliment considéré ne relève pas de la pédiatrie nutritionnelle mais du devoir sacré d'assistance des cadets aux aînés, sujet empreint de gravité morale s'il en est. On sera donc enclins à relier les choix ici faits de la langue nourricière et reçue des Pères et du caractère lignager et pour ainsi dire ethnique du sujet, dont l'association nous place dans le sillage d'un moraliste du siècle précédent, l'éminent poète Pèir de Garros (17). Ce faisant on soupçonne la portée morale de n'être pas exempte de métaphore politique de circonstance : si l'homme de bien ne peut abandonner les siens ni en oublier la langue, comment pourrait-il en trahir les lois? La fidélité civile fournit ainsi le ciment d'un honnête homme gascon.

Cette thématique se poursuivra d'ailleurs dans des œuvres occitanes postérieures, 
et semblablement dans notre cinquième livre, Lexhortation E remedes aulx desbauchés $\mathcal{E}$ dissolus, qui paraît prolonger le thème en l'inversant. En effet, la débauche est inévitablement allusive aux gaspillages et dilapidations, dont les plus choquants sont ceux du fils prodigue ; la dissolution étant naturellement évocatrice de mœurs générant la ruine des liens de solidarité familiale. Il semble donc qu'il faille envisager ces deux livres comme une sorte de diptyque traitant successivement des devoirs des (bons) enfants envers leurs parents et exhortant, avec une volonté édificatrice manifeste, les débauchés à remédier à leur travers. Passant de l'échelle de la famille à celle du peuple, cette métaphore politique peut-elle viser la tiédeur de certains Bayonnais vis-à-vis de l'institution municipale désormais soumise à l'étau centralisateur français, voire même de certains Béarnais à l'endroit de leur règne menacé ? On voit en tout cas que le dialogue ouvert par la conversation civile s'avère fertile - et que la cohérence intellectuelle sinon littéraire de notre ouvre sans titre est vaillamment soutenue.

C'est cependant la dimension morale, davantage que politique, que paraissent cibler les trois stances emphatiquement proclamées en vers pour mieux les souligner gascons. Laccent est ainsi placé sur le choix d'une forme poétique ordinairement exigeante dans la facture et élevée pour l'inspiration. Nous ne saurons peut-être jamais rien du style de celles-ci, mais on verra volontiers dans la véhémence des 'contra' répétés un indice de quelque chaleur lyrique. Il semble que l'auteur ait voulu, avec les vices dénoncés dans les deux dernières pièces: Yvrognes, gourmands et prodigues (cette fois nommément désignés), et : Pipeurs [tricheurs] et mauvais joueurs, dessiner comme un portrait particulier des catégories de vices auxquels l'exhortation précédente offrait remède, comme si succédait à un plaidoyer raisonneur et apologétique une galerie de caricatures satiriques et corrosives. S'agissait-il, castigat ridendo mores, de pièces satiriques à l'ancienne mode (Desportes, Régnier) ou de parodies burlesques baroques, dont la vogue ne cessait alors de croître?

Le terme de stances paraît d'abord l'exclure, a fortiori chez un moraliste. Non que la veine parodique ait ignoré la subversion de cette forme, comme d'autres genres. Mais l'usage réclame dans ce cas l'adjonction des spécifiants 'satiriques' ou 'burlesques', indication ici manquante d'un usage à contre-emploi du genre ou de la forme concernés. En effet que servirait de notifier le genre lorsqu'il s'agit de son exact inverse dans le registre des valeurs littéraires, son contre genre satirique? Il nous faut d'ailleurs tenir un compte plus attentif du premier vice, dans la stance sur les blasphémateurs. S’il ne s'agissait que des jurons, on pourrait considérer que l'usage en est indissociable de débauche et dissolution et ranger le blasphème au rang des intempérances de bouche et de jeu. C'est l'attitude des magistrats bayonnais qui répriment activement les jeux d'argent, particulièrement de dés, au motif « des blasphèmes qui s'y font ». La pratique juridique béarnaise est même plus rigoureuse : les Fors, code juridique de l'Etat béarnais, placent le blasphème sur le même degré de gravité que les coups et blessures volontaires d'une part, que la diffamation de l'autre, moyens également déloyaux et criminels de règlement des différents. Le blasphème est en somme déloyauté de la créature envers le Créateur. Il est vrai que les Fors punissent aussi du pilori la tromperie au jeu, le reste relevant de la moquerie publique (et les pasteurs réformés eux-mêmes ne manqueront pas de déplorer les débordements domestiques). Mais ne s'agit-il que de cela?

Lintensité des débats confessionnels dans l'Aquitaine de ce premier XVII ${ }^{\text {eme }}$ siècle oblige évidemment à mettre au premier plan l'aspect religieux, impie, du blasphème. Théophile de Viau n'allait pas tarder à en éprouver quelque chose. D'ailleurs le caractère blasphématoire résulte d'abord d'une perversion d'opinion, avant l'emploi des mots. Et c'est ici au sort de 
Vanini, dont les cendres sont encore chaudes dans la Toulouse de l'Interprect, qu'on doit penser. Papistes toulousains et Parpaillots béarnais rivalisent de rigueur dans la répression de ce vice, injurieux au Créateur autant qu'aux fidèles, subversif de la religion autant que du siècle, et ainsi d'une gravité institutionnelle majeure. Mais l'austère Bayonne, dans la tentative incessante d'améliorer la sécurité juridique de ses pratiques, n'est pas en reste en fait de morale, qui institue dès 1580 dans le corps de ville, une commission de trois magistrats inspecteurs chargé de son contrôle public. D'où vient dès lors l'exorbitant emploi de Pour en faveur du blasphème ? Cette badinerie du 'pour et contre' renouvelée des blasons controversistes du siècle précédent, pouvait-elle être innocemment de mise sur un sujet si peu badin, alors que la réaction anti-libertine atteignait une virulence exacerbée? Le ton général de l'ouvrage entrevu exclut pourtant une quelconque provocation libertine envers les dévots, sous le regard d'un évêque rigoriste.

Sans doute faut - il mettre cette dualité de polarités pour et contre au rang du dialogisme ouvert par la Civile conversation. S'il n'est pas badin, le pour n'est sans doute pas non plus sérieusement défendu, mais allégué tactiquement pour rendre plus séduisante la rhétorique défenderesse du contre, au final maîtresse du terrain. Il est possible que cette concession résulte de la conception extensive du blasphème pratiquée par le corps de ville, qui réprime aussi sous ce chef le port de masques, notamment lors des débordements du Carnaval. Voltoire tenterait ainsi une sorte de médiation visant à éviter l'exclusion complète du populaire du discours public. Dans ce formalisme de la dispositio, le pour et le contre rejoignaient le conventionnalisme désuet des joutes scolaires et des puys poétiques, mais non la spéculation contestataire des futurs 'libertins érudits'. De tels exercices étaient-ils présents à l'esprit de l'auteur? La production édifiante d'Eustorg de Beaulieu devait être bien oubliée, malgré son séjour à Lectoure. A défaut de puys, la Gascogne a bel et bien connu tant des pastorales dramatiques à sujets moraux (communes aussi en Pays Basque et Aragon) que des ouvrages de controverse en vers (18).

A y bien regarder, les quelques lignes confiées à l'énigmatique feuillet soulèvent (à défaut d'en résoudre) de bien intéressantes questions sur l'activité éditoriale et nous plongent dans une épaisseur contextuelle et surtout intertextuelle d'une consistance que l'on n'attendait pas. A défaut de retrouver l'ouvrage perdu, comme l'escomptait Gustave Brunet, misant avec optimisme sur la relative épaisseur du volume dont nous venons de préciser la physionomie (pour notre part, nous appréhendons plutôt la fragilité d'un format oblong de si médiocre hauteur, mais que savons-nous de la forme matérielle sous laquelle l'œuvre perdue s'est vraiment concrétisée ?), il sera donc intéressant d'essayer de voir quel apport peut être le sien dans la connaissance de l'institution littéraire occitane.

Le constat implicite d'une cuvre perdue, fugitivement répété depuis G. Brunet, est donc solidement explicité. Il emporte une conséquence encore moins examinée et pourtant nécessaire : la question de son auteur. Identification que la sèche rigueur structuraliste eut naguère tenue pour oiseuse mais que l'expérience montre essentielle pour une correcte approche, réaliste, de la littérature occitane, compte tenu du matériau linguistique dialectalisé et du déploiement particulier de l'institution littéraire dans un ensemble multirégional à seule polarité dominante exogène. Car au-delà du degré de plus ou moins grande connaissance 
prosopographique d'un auteur - et la biographie de celui-ci nous échappe - ce sont les fonctionnalités dégagées par ce que nous induisons de son insertion dans cette littérature et ses processus de développement, qui nous intéressent comme contribution à l'histoire de la communauté occitane.

Faute de témoignage externe corrélable à sa teneur rédactionnelle, l'auteur de l'ouvrage décrit par notre table 'flottante' est directement inconnaissable. Il nous reste à tenter de croiser à nouveau les approches substitutives déjà mises en œuvre, à partir de la matérialité éditoriale et à partir du contexte. S'agissant du cahier final signé *, le seul élément matériel directement apparenté est naturellement le reste du volume de L'Interprect et spécialement la mention au titre du nom de son auteur. En l'absence de source documentaire renseignant celui-ci, on doit donc relever les indices que ses propres ouvrages peuvent fournir sur son compte. Tous ceux qui ont consulté ce guide trilingue ont relevé la finalité commerciale des exemples conversationnels proposés ainsi que le souci d'explicitation grammaticale qui s'y manifeste, conduisant G. Brunet à voir en Voltoire quelque maître de langue. Mais si l'on veut approcher la personnalité de l'auteur, ces modèles phraséologiques stéréotypes ne sauraient être aussi instructifs que les deux cent pages de considérations variées sur la pratique du négoce qu'il développait dès 1607 dans son autre ouvrage Le Marchand (19).

Malheureusement l'attention s'est très longtemps limitée à la seule liste de proverbes gascons placée à la fin de ce dernier, comme si l'activité professionnelle de l'auteur avait paru négligeable au regard de l'intérêt parémiologique de cet appendice, seul digne de la considération savante et lettrée. Mais s'il existe chez Voltoire une coupure entre activité négociante et écriture gnomique (encore que les maximes du négoce et les sentences des proverbes eussent plus d'un point commun), ce n'est pas celle de ce préjugé de l'érudition positiviste. C'est que loin d'être issus d'une persévérante collecte personnelle résultant de ses liens concrets avec l'activité commerciale et les milieux humains basques ou aquitains, péninsulaires ou continentaux qui la pratiquaient autour de lui, les proverbes produits proviennent pour l'essentiel de... la traduction gasconne d'un recueil antérieur de large notoriété publié en français par le polygraphe hainuyer ou flamand Gabriel Meurier (20)!

Comme le fait observer Jacques Poumarède, historien des mours économiques de la Gascogne moderne, le Marchand est une véritable 'Défense et illustration' du commerce, voulant par ces termes signifier la pluralité d'approches et diversité de présentation du sujet par l'auteur. Il en suit et commente avec soin le déploiement (21), à partir de la dédicace 'Aux honorables et vertueux seigneurs, les juges, prieurs, consuls, bourgeois et marchands des cours et bourses communes establies par le Roy'. Les étapes de cette démarche sont expressément glosées par le titre, qui se présente davantage comme une énumération que comme unique enseigne technique ou littéraire (cf. note 18). Il y est d'abord fait mention, contrôlable à l'intérieur du volume par leurs titres de départ respectifs, de quatre parties annoncées avec luxe de détails, puis d'une adresse et d'un dialogue singulièrement mentionnés en dernier comme précédant 'le tout'. Cette disposition rétroactive tend malheureusement à passer inaperçue par suite de l'emploi peu explicite du mot sous au sens d'infra : 'Le tout ... disposé soubs l'Adresse', puis celui du mot 'invention' pour signifier, conformément à la terminologie rhétorique, la nature d'œuvre de fiction du dialogue : 'disposé sous l'adresse Ė invention de ... en forme de dialogue'. En effet, le double sens du mot adresse : 'avis, appel' (ici au lecteur), mais aussi 'habileté, art, ingéniosité', précédant le mot invention (ici sémantiquement proche de la seconde acception) employé cette fois sans article génère une lecture soudée (en syntagme figé) des mots 'adresse 
\& invention' dont l'apparente cohérence est en réalité fallacieuse. On doit en fait comprendre que les quatre parties d'abord citées comme 'un tout' sont, dans l'ouvrage, 'produites et disposées après l'Adresse faite en premier lieu au lecteur, puis la fiction en forme de dialogue à celle-ci consécutive', qui paraît viser un public plus idéel.

Ainsi rétabli dans l'ordre du contenu, cet intitulé disert marque la distinction introduite entre trois lots de pièces d'enjeux différents. D'un côté sont deux discours en prose, respectivement 'traictant des propriétez du négoce, de la qualité et condition du bourgeois et autres particularitez' (pages 50 à 80), et des causes 'de la décadence du négoce' (pages 81 à 108), réflexions d'expert manifestement citées en premier comme constituant le cour du sujet (« une instruction à la jeunesse pour s'advancer et maintenir dans le commerce », selon les termes de la dédicace). De l'autre suivent des appendices. C'est ce que traduit l'alternance des deux participes présents successifs : le premier référant au thème du marchand, caractérisé dans son activité archétypale 'le marchand traictant... du... négoce', le second référant exclusivement au volume placé entre les mains du lecteur 'contenant aussi un recueil de...', référent que la formule éditoriale alors d'usage consacré étend au dernier appendice '[contenant] ... ensemble les Motets gascons'. Lon comprend mieux ainsi la syntaxe initiale un peu lourde présentant les discours, constituée de quatre éléments : un titre, un sous-titre et deux parties ('Loffice du Marchand, ouvrage traitant du négoce, à savoir : de la qualité du bourgeois et marchand, des causes de la décadence du négoce').

Le premier discours est un plaidoyer que nous qualifierions aujourd'hui de corporatiste sur l'utilité sociale des métiers du commerce, « de l'intelligence, du fruict et labeur d'iceux ». Sa démonstration tend à assigner à ces acteurs économiques une situation exactement médiane dans le corps social de leur temps, équidistante des ordres privilégiés (clergé et noblesse, partie « subjecte aux grand vents des affaires mondaines pour sa hauteur ») et du tiers état laborieux. En effet celui-ci reste sujet « aux terreurs et débordements des affaires des lieux bas, mal disposé pour sa bassesse ou ignorance », formule qui garde probablement la mémoire des troubles sociaux bayonnais de 1573, à l'occasion desquels le corps de ville craignait en métaphores identiques « que de ces allumettes sortit un plus grand feu, lequel ne pourroit estre estaint » (cité par J. Pontet, op. cit. en note 13, p. 123). Voltoire reflète ici parfaitement la hiérarchisation du pouvoir entre les classes bourgeoises bayonnaises rivales pour le contrôle de la cité, et l'avènement de la suprématie politique des négociants parvenus à reléguer les fabricants, triomphe de la profession sur le métier. Aussi ne faut-il pas se méprendre sur sa revendication d'une "médiocrité que les philosophes louent et estiment si doctement » mais davantage relever sa connaissance et l'instrumentalisation consécutive de philosophes d'un profil qu'on devine réaliste. Faut-il voir là une trace janséniste, alors que séjourne à Bayonne de 1609 jusqu'à 1614, le jeune Flamand Cornélius Jansen, futur évêque de la métropole drapière d'Ypres, auprès de son fameux disciple Jean Ambroise Duvergier de Hauranne, futur abbé de Saint Cyran, mais enfant du pays, c'est-à-dire de son élite bourgeoise?

Cette mediocritas commerçante, reprise de sources italiennes et espagnoles développées depuis le Moyen Age dont il pouvait bien connaître le fonds par ses voyages, pétrie de savoirs théoriques et compétences pratiques (géographiques, marchands, arithmétiques, comptables, financiers, boursiers, mais aussi comportementaux, moraux, voire sociaux) largement attestés dans le texte, sous-tend une idéologie économique du bien public, basée sur l'échange déjà mondialisé et la libre entreprise, ne devant de compte qu'à Dieu et au Souverain. Un siècle plus tard, les intendants seront étonnés des fortunes ainsi constituées, jugeant les négociants 
de Bayonne «plus hardis et plus entreprenants » et son commerce « fort au dessus de celui de Bordeaux ». Voltoire promeut une vision émancipée de sa classe sociale " apte et propre à l'administration et gouvernement [des cités] » (il fait grand cas de celles de Bordeaux et Toulouse, et de leurs institutions municipales) aux antipodes des préjugés cléricaux à l'encontre du négoce et surtout de l'arbitraire arrogant des pouvoirs nobiliaires. Le mépris de cet Ordre pour l'activité mercantile (qui en explique la sous représentation dans la cité portuaire) semble bien le contre-modèle inconscient de sa modestie conquérante. Il est remarquable d'observer la coïncidence avec les années où l'aspiration à établir la dignité du corps de ville se fait la plus insistante, enjoignant aux édiles (également investis de fonctions juridiques) d'assister aux séances en tenue (robe et chaperon) au motif même de «tenir la modestie et décence de vie du magistrat » (cité par J. Pontet, op. cit., note 13, p. 97).

Le second discours est d'une tonalité toute inverse, qui se livre à une longue déploration sur la conjoncture récessive de la fin du XVI İme siècle. En observant les difficultés économiques qui frappent alors l'ouest occitan, Voltoire se révèle un témoin (objectif ou subjectif) d'une époque de prospérité antérieure, qu'on peut à la suite de J. Poumarède situer vers 1570 . Nous aurions donc affaire à un négociant d'âge et d'expérience, à l'heure de son bilan professionnel, et de possibles loisirs. Ces discours en français sont flanqués de deux appendices de caractère tout aussi positif à cette époque puisqu'apparentés aux loci communes par leur nature de 'recueils' compilant des exemples : l'un de 'certaines Similitudes ou considérations' (pages 109 à 128) observations à portée sentencieuse (22), l'autre des fameux Moutets guascous (pages 129 ter à 195) dits 'sentences récréatives'. Cette dernière acception, qui ne leur retire aucunement la qualité d'œuvre sérieuse, indique plutôt leur conception utilement instructive, vulgarisant une sagesse sinon une jurisprudence coutumières mémorisables et sécurisantes. Cette orientation perdurera dans le très circonstancié Interprect : "Considère que ce que j'en ay fait est pour le bien et commodité du public (...) estimant du moins que Dieu congnoistra [là] mon intention et volonté » (dédicace Au Lecteur).

Mais l'ensemble réuni pour Le Marchand paraît poser à l'auteur un problème de présentation au public : il lui faut pour le 'produire' devant ce dernier (nous disons aujourd'hui 'le publier') interpeller le lecteur par une adresse ajoutée à la dédicace aux pairs, et faire offre d'une fiction de voyage. Celles-ci apparaissent donc comme un troisième lot d'écriture, de prolégomènes nécessaires à la lecture, sorte de seuil éthique ou d'exergue subjectif. En effet, le caractère en est nettement personnel par le thème, qui est un voyage d'initiation (et nullement initiatique, puisque d'un négociant de grand chemin rompu au métier acceptant d'initier un jeune marchant débutant, qui a part congrue au dialogue), et surtout par l'aveu de paternité : Dialogue de l'Autheur et du Marchand (ce dernier étant l'apprenti). Et il s'inscrit doublement dans la littérarité puisque le dialogue est versifié, composé de 1144 vers, et par là évocateur des récits de voyage mythiques des Anciens, sinon précurseur du Télémaque de Fénélon par son prosaïsme tout contemporain. Le corps didactique de l'ouvrage est ainsi enserré entre les rimes gasconnes des proverbes finaux et l'embarquement narratif pour la navigation commerciale d'un vaste poème français. La diversité de ses fragments l'apparente à l'esthétique du discontinu prisée des moralistes, la cohérence gisant dans la visée. Il est aisé retrouver dans ces ordonnancements calculés et cette propension littéraire les caractères entrevus dans l'œuvre perdue de la table 'flottante'. Voltoire est manifestement l'auteur de l'ouvrage perdu, qui lisait aussi les philosophes.

On comprendra par conséquent que nous ne partagions pas les jugements très restrictifs 
portés sur Voltoire par Gabriel Clavelier : « le fastidieux récit du voyage » puis « les deux soporifiques discours » (dont J. Poumarède montre au contraire l'intérêt pour les idées et l'histoire économiques), et cette estocade : «Il n'est que de lire sa prose ou ses vers, car il s'est parfois hasardé à cet exercice, pour se convaincre qu'il fut, ainsi qu'il l'avoue lui-même, un esprit peu cultivé » (23). Il nous semble qu'il y a là confusion grossière entre culture (celle de Voltoire est même étendue) et savoir spécialisé : la formation lettrée dont cet autodidacte se dit frustré " pour y avoir été laissé inculte au milieu de ses ans », que nous comprenons ceux de sa carrière active. Détail qui laisse entendre une première initiation juvénile et le goût de renouer, dans des termes entre temps un peu vieillis, avec la grammaire et la rhétorique. Un collège avait été ouvert en 1571 par les soins des magistrats bayonnais, qui en surveilleront directement le développement, dont le régent assurait dès 1572 l'enseignement des humanités avec le de Officiis de Cicéron.

Certes le négociant reconnaît ses difficultés « non seulement pour la traduction mais aussi pour l'orthographe, ponctuation et autre particularitez » d'ailleurs en pleine évolution entre Pelletier du Mans et Malherbe. Mais il entend surmonter cet handicap culturel sans s'embarrasser de pédanterie: " De tels travaux sont du ressort de personnages fort capables et experimentez en ces affaires traductifs et interprétatifs ». Cette sagesse, comme sa curiosité, sont à la vérité tout semblables à celles d'un autre Gascon fils de commerçants venus d'Ibérie, Michel Eyquem, seigneur de Montagne et magistrat de Bordeaux. Où, mieux que dans les Essais dont le succès n'était plus à faire, Voltoire (qui avait peut-être approché le magistrat philosophe sur les quais du Port de la Lune) aurait-il trouvé plus docte estime de la modestie? (24).

S'il est certain que la versification de Voltoire n'a rien d'un Viau ou d'un Larade elle n'est point inférieure à celle de ses autres voisins Auger Gaillard ou Bernard Du Poey, encore moins de plus connus en meilleur lieu, Marc de Maillet ou Louis de Neuf-Germain en français. Il sait composer judicieusement, mais s'excuse de son « rude ramage, comme sortant d'un lieu infertile ». Il convient de ne pas se laisser abuser par ce tableau qui est un lieu commun des intellectuels Gascons de la Renaissance, déjà exprimé avec une poignante force poétique par Pèir de Garros (25). Aussi nous garderons-nous de lui dénier, avec J. Poumarède trop influencé par Clavelier, "la capacité intellectuelle de faire œuvre littéraire ». Il reste heureusement quelques strapontins au Parnasse ! Néanmoins la maîtrise du français, d'ailleurs en phase de mutation, a pu lui créer difficulté dans la poétique et la versification : on ne naît pas poète dans la langue étrangère (26). Et plutôt que nous fier au Dialogue, dont l'esthétique ni l'éthique n'orientent au lyrisme, nous éviterons le procès de sa versification jugée dans cette seule langue (27). On regrettera d'autant les pièces gasconnes perdues, davantage situées dans le prolongement polymorphe du Marchand qu'en lien avec un guide 'de poche' spécialisé.

Les deux spécimens qui précèdent les Moutets par exemple, soit un envoi Aus legidous, de quatre quatrains en rimes croisées, et un argument Aus Moutets, de 46 vers de strophes variables, dans lesquels il destine ses proverbes aux enfants (notamment ceux des marchands: aus hillets / Deus bourgés et marchans, aus que soun besiadets), laissent une impression de simplicité plaisante. On relève au passage l'intention didactique de ces versifications récréatives. Elle semble la clef du choix de la langue : non mouvement de curiosité envers l'originalité de la 'création populaire', mais mise à disposition sous une forme attrayante, linguistiquement familière et mémorisable de rudiments de sagesse comportementale, valables sous toutes latitudes. C'en est même le seul choix, puisque le corpus est d'emprunt; la qualité formelle, 
sans être travaillée pour elle-même, n'y est donc pas indifférente (28). En somme, quoi qu'humblement terre à terre, nous avons affaire à une tentative de prolongement sécularisée de la nationalisation linguistique de l'institution littéraire que le poète Arnaud de Salette avait offerte avec éclat aux Protestants de Béarn, par une autre traduction, deux décennies plus tôt (29). Cette veine éducative est évidemment fort éloignée de l'orgueilleux éclat du registre aristocratisant dominant alors les Lettres, y compris occitanes à l'ouest. Aussi peut-on, avec l'historien économiste, s'interroger sur la moindre légitimation socio-esthétique de cette production, cause probable d'une audience littéraire rapidement réduite.

Fidèle à ses principes, Voltoire paie devant la postérité son choix d'une médiocrité utilitaire d'inspiration humaniste, préférée à l'ostentation de la littérature dominante où le baroque appelle le classicisme. Ainsi se dégage, en creux, comme la figure d'un autre Auger Gaillard, soustrait au culte des Muses par ses navigations au long cours quand son aîné l'était par ses enrôlements partisans, s'essayant à la diversité mais ignorant la truculence d'un Libre gras semblablement perdu, dédaigneux de solliciter les mécènes royaux mais plus fier de son métier sinon de ses capitaux, moins ouvertement religieux mais développant des thèmes moraux et didactiques dont on a peu d'exemples occitans modernes, partageant cependant, bien qu'avec moins d'inquiétude, une forme de marginalisation de son art, et sa persévérance à l'illustrer. Comparaison, certes, n'est pas raison, mais il y faut ajouter le voisinage: dans les années 1585 le poète albigeois dans l'orbite de Du Bartas s'établit en Béarn où il publie ses derniers ouvrages, puis à Pau, touchant pension des Etats jusqu’à sa mort, en 1595.

Avons-nous ainsi poussé à ses limites rationnelles la connaissance du sujet ? Il est clair, une nouvelle fois, que 'la plume n'est pas tombée entre les mains de l'auteur occitan par hasard'. Sans avoir besoin de l'inventaire de sa bibliothèque pour en identifier les lectures, nous comprenons que Voltoire, s'il n'a pas fréquenté les letra-ferits moqués par Montaigne, est informé de façon globale du contexte idéologique, culturel et littéraire gascon de son temps. Il n'est donc pas dépourvu des moyens de sa tentative poursuivie sans tambour ni trompette. Il n'est pour s'en convaincre que d'observer à la suite de Jean-François Courouau (30), la concomitance en cette année 1607, dans la même ville de Toulouse, et qui plus est, dans le même atelier de Jacques et Raymond Colomiés, de l'édition du Catounet gascoun, quatrains gnomiques de Guillaume Ader (sur le modèle des Dysticha moribus attribués à Dyonisius Cato), des proverbes de Bertrand Larade (dans sa Muse piranese, d'après ceux de Baif) et des Moutets du Marchand, tirés de Meurier. La benoîte coïncidence serait étonnante dans la ville de Du Faur de Pybrac, auteur des fameux Quatrains français, au voisinage d'un des dernières gloires humanistes, Jules-César Scaliger, également amateur de proverbes, et alors qu'un concurrent des Colomiès vient d'y réimprimer Baif.

Ces faits ont conduit J.-F. Courouau à envisager l'excursus parémiologique de la Muse de Larade, toujours inquiet de sa notoriété, comme une 'réponse' hâtivement apportée à l'initiative de Voltoire. Sauf à y supposer une volonté extra-auctoriale, nous avouons quelque scepticisme devant cette hypothèse à cause du caractère peu valorisant tant de l'ajout de proverbes dans le recueil d'esthétique maniériste de l'exigeant sonnettiste, que de l'idée pour un impétrant aux Jeux Floraux de se défendre de l'initiative d'un marchand (ou il faudrait que la notoriété en eut été bien grande à l'ombre du Capitole). S'il faut certes que l'un ait précédé l'autre, pas 
plus avant qu'après ne s'impose l'avantage tiré par Larade sur la parution de Voltoire. Quelle qu'ait été la sensibilité d'amour-propre du premier, son public choisi ne s'assimilait en rien aux confrères auxquels s'adresse le mémento du négociant. La rivalité n'en étant guère à craindre avant, une jalousie de Larade n'aurait pu prendre corps qu'après la parution de sa Muse, mais le Commingeois jouissait alors par ses seuls vers (et ceux de sa Margalide de 1604) d'une suprématie sans appel.

Il nous paraîtrait à ce compte plus tentant de croire la Muse bayonnaise de 1620 émule de la Piranèse, moins par le fait d'une jalousie attardée du marchand que pour épouser une démarche esthétique commune, pour lui d'ornement à l'appui de son hommage corporatif ; ces Muses gasconnes elles-mêmes rejoignant la mémoire archétypale de celle de Du Bartas. Pour peu qu'il faille attribuer à la Muse de Voltoire une date plus ancienne, et à sa fréquentation de Toulouse une incidence littéraire, on pourrait esquisser un rapprochement avec le Recueil de divers poèmes de Jean Galaut, paru chez les mêmes Colomiés en 1611, où se succèdent de plaisants tableaux d'un joueur trop chanceux et d'un ivrogne amoureux. Mais il est certain, se contentât-on de l'attribuer à la mode ou à la demande supposée du public - la rivalité se ramenant alors à l'horizon d'attente' - qu'il y a là un indicateur manifeste de la consistance désormais atteinte par l'institution littéraire gasconne depuis le début de son 'Siècle d'or', en nombre d'acteurs, diversité de champs génériques, volume d'œuvres. Et corrélativement, en termes d'intertextualité comme on le devine ici, de circulation de textes comme on le voit si souvent. Cette courte exégèse en fait entrevoir un pan.

On objectera pourtant l'anonymat sous lequel furent si longtemps ensevelis le marchand et son œuvre: nous en avons esquissé une explication. Il n'est certes que trop vrai que l'auteur occitan en de très nombreuses occurrences ne se laisse que fantomatiquement entrevoir, faute le plus souvent d'une volonté entretenue d'en faire connaissance. Cette hypothèque, pour fâcheuse qu'en soit l'absence d'éclairage prosopographique et contextuel, est globalement peu préjudiciable pour une littérature nationale établie comme est la française, dont l'étude est elle-même formidablement institutionnalisée. Elle est bien plus gênante pour l'étude, combien marginale, d'une littérature dont la trame est sans cesse compénétrée par des éléments exogènes, dont le tissu doit incessamment être restauré pour prendre sens.

On aimerait donc en savoir plus sur Voltoire. Faut-il remonter aux sources généalogiques? On a évoqué les toponymes de Voltoyre en Aveyron et Cantal (31), mais ces attestations, anciennes ou nord-occitanes, sont encore plus répandues dans le Massif Central sous la forme méridionale Bouldouyre, Bouldoire, également bien attestée comme patronyme. Si l'on devait retenir cette origine foraine, il faut en effet penser à une hypercorrection graphique possible du phonème initial opérée en Béarn (le son [b] restitué par $<\mathrm{v}\rangle$ graphique). J.-C. Dinguirard a tenté une localisation linguistique, qu'il place en Gascogne intérieure, mais celle-ci se heurte au fort contexte bayonnais sous-jacent aussi bien au Marchand, qu'au dialecte basque du secteur de Saint-Jean-de-Luz et Ciboure choisi pour référence dans l'Interprect, et naturellement aux 'compatriots' assignés à la Muse. Et si le Marchand a circulé entre Béarn et Toulouse, il a pu aisément contaminer son parler de formes locales diverses selon ses relais. S'il fallait esquisser un hypothèse qui conciliât ces données, nous serions tenté de supposer une origine de la Haute Guyenne ou du sud-ouest du Massif occitan, où les corporations colporteuses et marchandes furent développées, et une migration, peut être sur deux générations, vers le bas Quercy puis la Gascogne, qui expliquerait le maintien de contacts Toulousains, voire vers Lyon. Mais c'est dans la région de Bayonne, son authentique port d'attache, qu'auraient probablement le plus 
de chance d'être retrouvées ses traces matérielles.

Une telle éventualité rendrait compte également de l'aptitude voire l'appétence de Voltoire pour la polyglossie, de son expérience consommée du commerce des hommes pour lequel son empathie paraît effectivement atavique, mais surtout de l'embarras esthético-linguistique qui semble entraver sa veine créatrice littéraire. Car le marchand paraît avoir pâti de cette difficulté particulière aux individus charnègues, ceux que la vie a placés à cheval sur plusieurs communautés sociolinguistiques et qui n'appartiennent univoquement à aucune. C'est le malaise que traduit si bien Montaigne, persiflant l'élégance 'mignarde' du français et admirant l'efficience 'mâle et militaire' du béarnais - langues également véhiculaires et royales -, mais irrépressiblement honteux de la sienne propre, cet occitan vernaculaire périgourdin 'brode, traînant et esfoiré' qu'il renie, intermédiaire informe et disgracié entre poitevin-saintongeais, limousin et gascon véhiculaires aux caractères bien marqués; tentant dérisoirement de nous faire accroire qu'un bon peu de latin en avait immunisé le petit Michel et son monde (32).

Mais dans l'attente d'en apprendre plus par une réponse interne à l'objection de l'effacement, n'est-on pas en droit de s'interroger sur de possibles traces externes laissées par cette production? Une démarche assez semblable permit de retrouver, rationnellement et non par hasard, en son pays même plutôt que dans l'histoire littéraire, l'œuvre perdu de François Rempnoux. Est-on certain que l'œuvre du marchand de Bayonne n'ait laissé aucune trace dans l'écriture ? Est-on si sûr que le dialogue versifié en gascon ait expiré sous sa plume ? On en doutera certainement en lisant les 2622 vers du Calvinisme de Bearn divisat en siex eclogues dialoguées de Jean-Henri Fondeville, ca 1693, opportunément réédités récemment (33). On y rencontre la même inscription des séquelles interminables des troubles religieux (l'œuvre tente de rallier au catholicisme les Protestants nicodémistes après la Révocation de l'édit de Nantes), la même veine didactique, les mêmes préoccupations morales dans le moule religieux, servies cette fois par une langue dynamique, ruisselante d'authenticité terrienne. La thématique des devoirs des pères et des fils se retrouve avec insistance dans la littérature béarnaise, tant avec La Pastourale deu paisan qui cerque mestiè a soun hilh du même auteur (34) que dans une Noubelle pastourale bearneze et des pièces en français. Léthique et l'esthétique jusqu'ici perçues comme isolées de l'œuvre importante de Fondeville deviennent beaucoup moins singulières dans le sillage de Voltoire, acquièrent une nouvelle profondeur, la perception socioculturelle que nous en retirons devient plus cohérente, et les perspectives d'interprétation se multiplient d'une production que la méconnaissance seule laissait croire monotone.

Certes, ce bout de péritexte soulève autant de questions qu'il n'en résout. Rarement pourtant si peu de lignes délivrent un si riche message. Avec celui-ci la production occitane moderne perd encore un peu de ce caractère accidentel et monstrueux ('patois', à valeur originellement adjective) que le processus de provincialisation lui assignait. Son étude quitte le 'cabinet des curiosités' pour entrer dans le concert de la littérature européenne dont l'historiographie cesse de reproduire le tableau de classement des règnes dominants et des seules langues que ceuxci emploient à élargir leurs empires. Puissent cette émancipation s'étendre à la notoriété de la littérature d'oc contemporaine et cette mise à jour profiter à son tour, malgré le défaut d'enseignement, à l'instruction de jeunes générations occitanes. Et à tous autres.

\section{NOTES}

1) Pour autant nombre d'observations en la matière sont à relever dans les travaux des occitanisants ces dernières décennies, sous les plumes de R. Lafont, Ph. Gardy, Jean-François 
Courouau, Jean Eygun, Pierre Escudé et autres. Pour la question des didascalies, approche dans : C. Bonnet, «Didascalies et signifiance : lectures du théâtre occitan préclassique », Revue d'Histoire du Théâtre, 2001, fasc. 4, pp. 265-274.

2 ) Xarles Videgain, éd. et prés. - L'Interprect, ou Traduction du françois, espagnol, et basque, de Voltoire... - [Réédition en fac simile de l'éd. de Lyon, A. Rouyer, 1620]. - [Pau] : Manutius (I.C.N. impr.), 2000. - [2]-XIV-[10]-280-[3] pages, $11 \mathrm{~cm}$. oblong. Voltoire, bourgeois et peut-être armateur bayonnais était déjà l'auteur d'un guide du négociant intitulé Le Marchant, que nous examinons plus bas.

3) S'agissant de l'exemplaire de la Bibliothèque Municipale de Bordeaux (B. 2.866 Rés.) dont j'avais relevé la description suivante lors de l'établissement par mes soins des notices des ouvrages anciens du Catalogue collectif des fonds occitans des Bibliothèques publiques de Bordeaux (Bordeaux, Société des Bibliophiles de Guyenne, 1993):

LINTERPRECT / Ou Traduction du François Efpagnol et Bafque de VOLTOIRE / CONTENANT / Plufieurs Parties ainfi qu'eft Contenu à la Table / Dernier efcripte. / [Petit ornement typogr.] / A LYON / PAR A. ROVYER Imprimeur du ROY. // - Petit in quarto oblong 8 x 12,5 cm., de [6] ff.-280 pages, signé A- $\mathrm{Z}^{4}$, Aa- $\mathrm{Nn}^{4}$, un cahier signé *2 (TABLE DE / LINTERPREST OU... ) inséré entre les deux premiers feuillets ( $\left[\mathrm{A}^{1}\right]$ : titre, et $\mathrm{A}^{2}$ : AV LECTEVR SALVT ), verso du titre blanc, un feuillet signé * ajouté à la fin ( TABLE DV CONTENV EN CE LIVRE), objet de la présente analyse.

Cette collation, que ne laisse pas forcément déduire l'édition en fac-similé, confirme par le parallélisme des signatures, le caractère superfétatoire de la seconde table. On ne peut déterminer si ce feuillet final était originellement constitutif d'un cahier de deux, la reliure actuelle n'ayant pas conservé les gardes primitives. Par ailleurs la dernière ligne de texte se présente décentrée vers la gauche (le mot Par est justifié imperceptiblement plus à gauche que le mot $\mathrm{Ou}$ de la deuxième ligne), le blanc disponible à droite étant occupé par une mention manuscrite du millésime 1620, elle-même justifiée faiblement plus à droite que la syllabe esdu mot espagnol des lignes 2-3, comme si un emplacement vierge avait été ménagé à cette fin au bout de la dernière ligne (car on ne voit pas de trace d'altération d'un quelconque millésime imprimé qui ait fait l'objet de substitution).

4) Nous suivons dans cette terminologie la distinction introduite par Gérard Genette (en dernier lieu Seuils, 1987) entre les éléments paratextuels internes présentant l'ouvrage rendu public (manuscrit ou imprimé), tels que titres, dédicaces, appendices, mentions auctoriales ou éditoriales (y compris, comme ici, dispositions typographiques), etc. en lien immédiat, constituant proprement son péritexte et ceux, externes, rédigés à propos du même ouvrage, en rapport médiat, constituant l'épitexte ; tous éléments contribuant par un lien ou rapport direct à déterminer socialement et instituer le texte et devant par conséquent être distingués du contexte, environnement global sans lien textuel direct. Lobjet textuel table ou sommaire des ouvrages anciens, pourtant souvent riche d'indices et d'informations, est demeuré jusqu'ici peu théorisé.

5) Principalement le bordelais Gustave Brunet (Anciens proverbes basques et gascons recueillis 
par Voltoire, Paris, 1845, éd. revue et augm. Bayonne, 1873 avec éd.) ; Pierre-Alexandre Gratet-Duplessis (Bibliographie parémiologique, Paris, 1847, avec éd. en appendice); Otto E. Moll (Sprichwörterbibliographie, Frankfurt a. Mein, 1958). Voltoire a néanmoins fait l'objet d'un regain d'intérêt de la critique depuis la seconde moitié du XX siècle: Gabriel Clavelier, «Le Plus ancien recueil de proverbes en langue d'oc : les Moutets gascous de Voltoire [joints à son traité de négoce Le Marchand, Toulouse 1607] », Annales du Midi, t. 48, 1936, pp. 2952 ; Jean-Claude Dinguirard, "'So ditz la gens anciana' : recherches sur les plus anciennes collections de proverbes gascons [Marcabru, Larade, Voltoire] », Via Domitia, t. 28, 1982, fasc. 2, pp. 3-108; Jacques Poumarède « Une 'Défense et illustation' du commerce par un marchand gascon sous le règne d'Henri IV [ le Marchand] », Hommage à Romuald Szramkiewicz [éd. par J.-L. Harouel, M.-B. Bruguière et J. Brouineau], Paris, LITEC, 1998, pp. 425-444 ; et naturellement Xarles Videgain, «Les proverbes en basque de Voltoire [ceux de l'Interprect] », Les Voix de la Nymphe aquitaine : écritures, langues et pouvoirs, 1550-1610 [éd. par J.-F. Courouau, J. Cubelier de Beynac, Ph. Gardy], Agen, Centre Matteo Bandello, 2005, pp. 153 169. Il est utilement replacé dans un cadre général dans l'ouvrage de Jean-François Courouau, 'Moun lengatge bèl' : Les choix linguistiques minoritaires en France, 1490-1660, Genève, Droz, 2008, pp. 124-128.

6) Cette particularité n'avait pas échappé au Bordelais Gustave Brunet, qui n'en tira cependant pas davantage parti en décrivant hâtivement le « feuillet qui donne l'indication d'un autre ouvrage de Voltoire », qu'il estime « assez gros pour avoir échappé aux chances de destruction » et « qu'il serait fort intéressant de découvrir » dans sa note: « Un Ecrivain gascon peu connu : Voltoire », Revue d'Aquitaine, t. 10, 1866, pp. 127-129.

7) Abraham Rouyer n'est guère documenté en dehors des produits de ses presses qui nous ont été conservés. Sa production durable et abondante à Orthez atteste de son adhésion à la Réforme. On peut envisager que les événements des années 1619-20 et les textes publiés par ses soins l'aient engagé à déguerpir. Le choix de Lyon s'expliquerait malgré l'éloignement s'il s'agit bien d'un membre de la célèbre dynastie d'imprimeurs fondée dans cette ville au siècle précédent par le lochois Guillaume Rouillé, documentée par Henri Baudrier au t. 9 de sa Bibliographie lyonnaise, et dans son étude sur les variations graphiques du nom familial (il faut tenir compte ici des spécificités phonologiques et orthographiques de l'occitan béarnais). Toutefois, A. Rouyer a été documenté depuis par Emile Dusolier («Vieux imprimeurs et libraires de Bordeaux », Bulletin des bibliophiles de Guyenne, 1939, pp. 122-123) dans des actes de 1610 et 1611 en qualité de "marchand libraire de la présente ville de Bordeaux, y demeurant en la rue Saint Jammes [Jacques], paroisse Saint Eloi », ville catholique mais mieux placée pour le commerce de librairie avec le Béarn (les Millanges ont la même démarche). Rouyer résidait-il à Bordeaux, se contentant d'exploiter des presses à Orthez ? La longue présence du typographe en Aquitaine et l'absence de concurrent à Bayonne peuvent expliquer la conjonction avec l'auteur (Voltoire avait précédemment publié à Toulouse) ou la commande d'un libraire local ; le transfert de l'atelier à Lyon aurait entraîné un éloignement réciproque propre à justifier quantité d'aléas. S'il reste possible que l'adresse de Lyon ait été fictive, dictée par la prudence de confondre ses productions parmi celles des homonymes de la place, on n'en voit guère, en l'espèce, la nécessité religieuse ni politique. 
8) Au terme d'une analyse géolinguistique J.-Cl. Dinguirard proposait, de façon inattendue au regard du basque et de Bayonne, de situer en Gascogne intérieure la langue de ces poèmes, op. cit. supra note 4 , pp. 54 sq.

9) Pour nous limiter à nos propres contributions dix-septiémistes, Mailhot, Rempnoux, La Rancontre des Bergers, Rousset. Plusieurs éditions récentes de manuscrits ont aussi restitué leur véritable nature à des œuvres rendues méconnaissables par une tradition dévalorisante (pour nous, L'Hausano de La Feuillade, tragi-comédie politique).

10) Voir les ouvrages de Bernard Beugnot, Le Discours de la retraite, au XVII e siècle : loin du monde, loin du bruit, Paris, P.U.F., 1996 et de Thomas Pavel, L'Art de l'éloignement : essai sur l'imagination classique, Paris, Gallimard, 1996 ; ceux sur la pastorale de Jean-Pierre van Elslande, L'Imaginaire pastoral du XVII ${ }^{e}$ s., Paris, P.U.F., 1999 et Stéphane Macé, L'Eden perdu : la pastorale dans la poésie française de l'âge baroque, Paris, H. Champion, 2002 ; ou sur un plan plus politique nos observations: François Rempnoux : la littérature occitane entre baroque et classique, Gardonne, Fédérop, 2001.

11) Francitan : terme proposé à la suite de Robert Lafont par l'école sociolinguistique occitane pour désigner des formes de langue mixte, soit employées délibérément à des fins burlesques, dans la littérature moderne (Guill. de Reboul, La Feuillade, Cortète de Prades, Viandasso, etc.), soit pratiquées à l'époque contemporaine suite à la généralisation de l'expression française, dans des milieux populaires non ou néo-francophones conservant phonologie, lexique et syntaxe occitans.

12) Guillaume de Salluste Du Bartas, Poème dressé pour l'accueil de la Royne de Navarre faisant son entrée à Nérac, auquel trois Nymphes débattent qui aura l'honneur de saluer sa Majesté, souvent réédité à la suite de la Judith ou des Euvres de cet auteur, jusqu'à nos jours (quelques ex. dans le Catalogue des fonds occitans de Bordeaux cité note 3, pp. 144-147). Voir Philippe Gardy, La Leçon de Nérac: Du Bartas et les poètes occitans, 1550-1650, Talence, Presses Universitaires de Bordeaux, 1998.

13) Pour l'étude du riche contexte bayonnais, nous nous sommes appuyés principalement sur l'Histoire de Bayonne rédigée sous la direction de Joëlle Pontet, Toulouse, Privat, 1991, «Univers de la France».

14) Voir les études réunis par Alain Montandon Pour une histoire des traités de savoir-vivre en Europe, Clermont-Ferrand, Université Blaise Pascal, 1995, en particulier celle de Claude Margolin, «La civilité nouvelle : de la notion de civilité à sa pratique et aux traités de civilité » et celle d'Emmanuel Bury. Sur le concept esthétique de vesiat/vesiadura (parent des 'bel usage' et 'je ne sais quoi' français contemporains), cf. Ph. Gardy « Le Goût de la beziaduro: d'une 'manière' toulousaine au XVII ${ }^{\text {ème }}$ siècle » repris dans La Leçon de Nérac (ouv. cité note 12), pp. 195-216. Encore que la civilité de Voltoire dût incliner davantage vers l'assimilation d'un autocontrôle social et des savoirs relationnels du commerce que vers la galanterie aristocratique. 
15) Les monarchomaques prônent une monarchie régulée par les Etats ( le corps politique « fait le roi », conformément à l'étymologie ), ce qui correspondait au type constitutionnel béarnais tel que l'avaient (a)ménagé les Fors. Le représentant le plus remarquable de ce parti était à ce moment crucial Jean-Paul de Lescun, seigneur de Piets. Voir son apologie sur Le Serment solemnel des seigneurs souverains de Béarn à leur nouvel advenement à la Seigneurie. Presté par le feu roy Henry le grand (...) l'an 1581. Avec les articles du for, E autres extraicts des registres des Estampes de ladite Souveraineté. Contre l'union du pays à la couronne de France, E la main levée des biens ecclésiastiques poursuivies par les evesques d'Oloron E de l'Escar, imprimée à la suite de sa Généalogie des seigneurs souverains de Béarn, empereurs, rois et autres princes qui en sont descendus, Paris, 1616 et tiré à part.

16) Notamment La Mouche [parlant] à l'Anti Moyne, sieur de Pieds, compilateur de la 'requeste genealogique', copiste de divers extraicts reformez, i. seditieux \& cornemainbasse, contre la main-leuee des biens Ecclesiastiques de Bearn, sans lieu ni date, et Les Défenses de Jean Paul de Lescun, seigneur de Pietz, conseiller du Roy en ses conseils ordinaire et cour souveraine de Bearn, conseil d'Estat et prive de Navarre, contre les impostures, faussetes \& calomnies publiées, tant contre le service du Roy et droits de sa souveraineté en Bearn, a la ruyne des Eglises reformees recueillies en ycelle, en general, qu'en particulier contre la personne \& Maison du defendeur, en deux libelles diffamatoires... A Orthes, par Abraham Rouyer, 1619. Le nom de l'éditeur ne saurait nous être indifférent. La compromission dans laquelle il se vit ainsi impliqué pourrait expliquer son brusque départ, réel ou fictif pour Lyon, devant la venue de Louis XIII. Tableau du contexte chez Thierry Issartel, "L'Etat et la tolérance religieuse en Béarn, 1599-1620 : théories catholiques et protestantes de la souveraineté », Paix des armes, paix des âmes [actes du Colloque international de Pau,1998], Paris, Société Henri IV, 2000, pp. 313-332. Vue générale chez Paul-A. Mellet, Les Traités monarchomaques : confusion des temps, résistance armée et monarchie parfaite, 1560-1600, Genève, Droz, 2007.

17) Notamment dans la célèbre épître versifiée jointe à ses Poesias de 1567 , où il remercie son interlocuteur de l'avoir suivi dans la défense de "la causa damnada / De nosta lenga mespresada »

(vers 5-22) :

Damnada la podètz enténer Si degun non la vòu dehéner.

O praube liatge abusat,

Digne d'ester despaïsat

Qui leishas per ingratitud

La lenga de ta noiritud,

E non hès conde de l'ajuda

Au pais naturau deguda:

Aquò b'es, a plan tot pensar

Son païs mau recompensar.
Vous la pouvez bien dire damnée

Si personne ne daigne la soutenir (...)

Oh, malheureux lignage dans l'erreur,

Digne d'être chassé de ton pays

Pour délaisser, par ton ingratitude,

La langue de ceux qui t'ont nourri (...)

Et ne fais cas de l'assistance

Due au pays de ta naissance!

Voilà vraiment, tout considéré,

Son pays bien mal récompenser.

Texte, en dernier lieu, chez J.-F. Courouau, Premiers combats pour la langue occitane: manifestes linguistiques occitans, XVI-XVII ${ }^{e}$ s., Biarritz, Atlantica, 2001, pp. 43-60. 
18) Voir l'ouvrage de Léonce Peyrègne sur Jean Cazaurang, de Lanne (Pyrénées Atl.), Cazaurang de L. et son adaptation de la pastorale 'Mardi-Gras', Pau, Marrimpouey jeune, 1978. Le terme ne désigne plus ici un théâtre nobiliaire dans le sillage de l'Astrée, mais une forme de comédie de mours rurale également pratiquée dans le nord-ouest de la péninsule. Les prétendues 'comédies' de Marguerite de Navarre en avaient promu les potentialités édifiantes. La Noubelle pastourale bearneze, 1761 et rééditions, parait traiter à travers l'allégorie biblique des douze fils du patriarche Jacob, de la question du départ des cadets (et du métier dont il leur faudra vivre) ou de leur maintien au service de l'ostau. La Pastourale deü paysaa de J.-H. de Fondeville, d'un traitement plus littéraire (influence moliéresque ?), en inverse la problématique, le père d'un garçon récusant trois zélateurs successifs de métiers jugés charlatanesques et les femmes de la maison chassant un quatrième, maître d'armes. Fondeville est aussi l'auteur d'un grand ouvrage de controverse 'pragmatique' (ou 'relationnelle') et non théologique (ou dogmatique), les églogues du Calvinisme de Bearn (cf. note 32).

19) LE / MARCHAND, / TRAICTANT DES / proprietez \& particularitez du com- /merce \& negoce. / De la qualité E condition du Bourgeois E Marchand, / auec certaine inftruction à la ieuneffe pour s'y / aduancer \& maintenir. / Du motif de la decadence, ou ce negoce ce void / maintenant reduict. / Contenant außi vn recueil de certaines Similitudes / ou con/iderations. / En emble les motets Ga cons, ou entences recreatives. / Le tout produict E difpofé Joubs l'adreffe E inuention / de fon embarquement \& voyage, en forme / de Dialogue. / [ornement typogr.] / A TOLOSE, / Par la Vefue de I. Colomiez, à l'en eigne du nom / de IESVS, Et Raym. Colomiez, Imprimeurs / ordinaires du Roy. / 1607. // Petit in 12. Le nom de l'auteur d'après la dédicace. [Sic pour l'accentuation et les espacements typographiques autour des virgules].

20) La démonstration en a été apportée en 1936 par Gabriel Clavelier, op. cit. note 5, qui a identifié cette source dans l'ouvrage de Gabriel Meurier primitivement paru sous le titre de Recueil des sentences notables, dicts, dictons communs, adages, proverbes et refrains, la plupart traduits du latin, italien et espagnol, Anvers, Jean Waesberghe, 1568, diffusé en France sous le titre de Trésor des sentences dorées, dicts, proverbes et dictons communs (sept éditions à Rouen, Lyon et Paris entre 1568 et 1660). Le procédé n'a alors rien de surprenant : son compatriote Larade emprunte au même moment moitié de ses Areproués gascous à Jean Antoine de Baif ( Mimes, Enseignements et Proverbes, 1576 pour le livre Premier, dont l'édition complète de 1597 est précisément rééditée à Toulouse, par J. Jagourt, en 1605) ; il entre dans une pratique de récriture maniériste, particulièrement développée dans les genres brefs, que justifie le succès de la production gnomique dans la seconde moitié du XVI e siècle. G. Clavelier explique le choix de Voltoire par la forme rimée ou du moins assonancée donnée à ses sentences par Meurier, relevant que les Moutets guascous répugnaient à renoncer aux formes rimées (distique, quatrain, plus rarement sixain). Cette propension versifiée en fait l'emblème sinon du pauvre du moins, on va le voir, du laborieux.

Il n'est pas sans intérêt de noter, au passage, que le second ouvrage de Voltoire, L'Interprect, peut également être mis en parallèle avec des sources flamandes maritimes, comme le Dictionario, coloqvios, o dialogos en quatro lengvas, flamenco, frances, español y italiano de même format in 16 oblong, paru à Anvers dans les mêmes années (1571).

21) Jacques Poumarède, «Une Défense et Illustration du commerce par un marchand Gascon, 
sous le règne d'Henri IV», art. cité note 5. Il est curieux que cet analyste judicieux n'ait pas cherché à corréler, à travers la corporation en objet, la vie du marchand Voltoire et l'activité du port de grand commerce maritime dont il représente à plusieurs égards une sorte de porteparole.

22) On ne peut qu'être frappé de trouver à nouveau son modèle possible en Meurier (vers 15301610), auteur du recueil La Perle des similitudes, non moins propre à gens de quelconque estat, condition et qualité que très convenable au grand avancement de la jeunesse et soulagement de la vieillesse, Malines, G. Cranenbroeck, 1583, et grand fournisseur à partir de 1548 d'ouvrages de linguistique et d'éducation, dont un guide bilingue pour l'enfance La Guirlande des jeunes filles en françois et flamen, Anvers, 1587. On ne peut écarter la possibilité que le Bayonnais ait rencontré l'Anversois dans ses navigations (Voltoire a notamment fréquenté l'Angleterre) et trouvé dans les langues et l'éducation un terrain d'échanges avec ce polygraphe.

23) Dans son étude de 1936 sur les proverbes (cf. supra notes 5 et 20), pp. 32-33.

24) On rapprochera naturellement la haute estime du Marchand pour les institutions municipales et le dynamisme commercial et maritime de Bordeaux, de l'activité déployée par Montagne (-taigne pour la francophonie) tant pour la sauvegarde de la liberté portuaire au milieu des troubles civils, notamment en mai 1585, que dans la mise à jour du coutumier, outil juridique régulateur de la cité, Las Coustumas de Bourdèu naturellement en occitan gascon. Voir notamment Montaigne maire de Bordeaux, Bordeaux, L'Horizon chimérique, 1992 ; Les Ecrivains et la politique dans le sud-ouest de la France autour des années 1580 [actes du colloque de Bordeaux, 1981], Talence, Presses Universitaires de Bordeaux, 1982, notamment les communications de Michel Péronnet, André Tournon et Arlette Jouanna ; André Tournon,

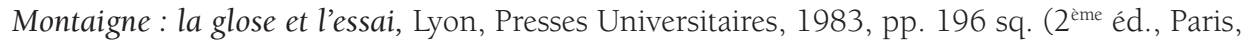
Champion, 2000, $\mathrm{id}^{\circ}$ ). Au titre de la curiosité de ce dernier, on peut ajouter son évocation (sur quelle source?) de telle étrange coiffure féminine bayonnaise dans l'essai III, 5, Sur des vers de Virgile - où il s'excuse de sa liberté en protestant précisément « J'ayme la modestie ».

25) André Berry, L'ouvre de Pey de Garros, poète gascon du XVI ème siècle, Talence, Presses Universitaires de Bordeaux, 1997 ; voir aussi Robert Lafont, Renaissance du Sud, Paris, Gallimard, 1969, ainsi que les actes des colloques d'Auch, 1965: Pey de Garros et son temps, Toulouse, Institut d'Etudes Occitanes, 1968, et Lectoure, 1981 : Pey de Garros, ca. 1525-1583, Béziers, C.E.L.O., 1988. Il n'est jusqu'à Montaigne qui n'entonne le regret d'écrire « chez moy, en pays sauvage, où personne ne m'ayde ni me relève [redresse mes fautes], où je ne hante homme qui entende le latin - et du français [encore] un peu moins » Essais, III, 5.

26) Françoise Bériac relève (Histoire de Bayonne, ouv. cité note 13, page 74) qu'en dépit de la conquête française en 1451, les élites bayonnaises ne montrent « pas la moindre trace de francophonie » au seuil du XVI ${ }^{\text {ème }}$ siècle et recourent à des interprètes - au truchement de Voltoire en somme. Dans la relation de son voyage en Espagne (1679), la comtesse d'Aulnoy découvre, à l'instar de Racine à Uzès, que les dames de la bonne société de Bayonne parlent toujours gascon (les maris avaient-ils profité des leçons du négoce ?). Pour la difficulté de la création littéraire dans l'autre langue, voyez Auger Galhard, 1579: « Mas en fransez ieu ny sabi 
pas gaire / Millou parli la lengo de mon paire " 'Pour le français, je ne m'y connais guère, / Je parle mieux la langue de mon père' (EEuvres, éd. Ernest Nègre, 1970, pp. 48-51). Montaigne exprime clairement cette difficulté : « J'ay toujours une idée et certaine image trouble en l'ame qui me présente, comme en songe, une meilleure forme que celle que j'ay mis en besongne; mais je ne la puis saisir et exploiter. Et cette idée mesme n'est que du moyen estage. [...] Tout est grossier chez moi ; il y a faute de gentillesse et de beauté. [... Mon style est] plustost grave et sévère - au moins si je dois nommer stile un parler informe et sans regle, un jargon populaire, et un proceder [développement] sans definition, sans partition, sans conclusion, trouble (...)», comme dans la dévalorisation de son occitan: «Mon langage [expression] françois est altéré en la prononciation et ailleurs [pour le reste] par la barbarie de mon cru [origine]. Je ne vis jamais homme des contrées de deçà [en deçà de la Loire: les pays d'oc] qui ne sentît bien evidemment son ramage [...] », Essais, II, 16, De la praesumption. On note la similitude de termes. Voir encore note 31 ci après.

27 ) Jacques Poumarède, art. cité note 5, page 433, sévérité paradoxale pour une médiocrité (des alexandrins boiteux, l'hémistiche hasardeuse, un manque de figures mythologiques qui pourtant 'encombrent' ailleurs) fort éloignée de l'incapacité alléguée, aggravée de lacune littéraire : le dialogue, même versifié, notamment pastoral, n'est certainement pas en désuétude entre le XV ème s. et Fontenelle ... mais bien la source utilisée (Littérature française de Godefroy, 1878 !). Sur l'expansion du dialogue (y compris les traités: Sigonio, Speroni, Le Tasse) et l'extension du dialogisme (dont les manuels de grammaire ou de langues) à ce moment, voir par ex. les études d'Eva Kushner, Le Dialogue à la Renaissance: histoire et poétique, Genève, Droz, 2004 ; celles réunies sous la dir. d’Anne-Marie Chabrolle-Cerretini et de Véronique Zaercher, Dialogue et intertextualité : Europe, XVI-XVII ${ }^{e}$ s., Sarreguemines, éd. Université de Nancy II, 2005 ; ou de Roger Friedlein, El Diálogo renacentista en la península ibérica, Stuttgart, Steiner, 2005.

28) On peut ainsi se demander par ex. si le terme moutet/motet (diminutif occitan de mout/ mot, au sémantisme initial lexical de 'mot émis, locution') employé préférentiellement à reproèr par Voltoire, n'inclut pas ici une trace des fonctions rhétorique et musicale du motet comme clausule donnant lieu à des effets de rythme et d'assonance, tels que peuvent le traduire 'formule, formulette'. Cf. Pèire Godolin: "L'Angel nos a dit un grand mot / Lausat siá Diu per dessus tot " 'un grand principe'; et "Qui son les que s'i fan à cantar le motet / A l'aunor del bèl Enfantet ? "qui répètent le motet' [apprennent un air de Noël], allusion aux enfants de manécanterie (nous citons d'après la récente édition de ses Euvres par Pierre Escudé, Toulouse, Privat, 2009, noëls, pp. 281 et 366). Ces moutets pour enfants pourraient bien avoir eu une visée scolaire dans le sillage de Meurier.

29) Arnaud de Salette, ministre, Los Psalmes de David metuts en rima bernesa, 1583. Ed. navèra par Robèrt Darrigrand, Orthez, Per Noste, 1983 et nouvelle éd. complétée (avec introduction historique par Philippe Chareyre) Paris, Champion, 2010. On n'aura garde de négliger la portée stylistique et esthétique de la réflexion parémiologique en langue maternelle, comme nous l'avons relevé à propos de Grégoire Mailhot («G. M. et la littérature satirique occitane à l'époque classique : une page limousine inconnue, 1700 », Bullet. de la Soc. Archéol. et Histor. du Limousin, t. 126, 1998, pp. 101-142, et pour son recueil : Louis Bonnaud, « Un Recueil de 
proverbes limousins en $1710 »$, idem, t. 97, 1970, pp. 203-206).

30) J.-F. Courouau, Moun lengatge bèl, 2008, op. cit. en note 5, pp. 133-134.

31) J. Poumarède, "Une 'Défense et illustration' du commerce... », art. cité en note 4, p. 427.

32) Montaigne, Essais, II, 16, De la praesumption (cf. supra, note 26) n'innove pas, l'idée est déjà sous jacente chez l'Angoumoisin François de Corlieu. Voir sur cet aspect, après celles de R. Lafont et F. Garavini, nos observations « Entre vernaculaire et véhiculaire : l'écrit occitan à l'époque moderne dans les confins nord-ouest du domaine linguistique », Aguiaine, t. 27, 1995, pp. 17-32 (actes du IV colloque de la SEFCO, Saintes, 1993). En outre, la dédicace de Voltoire Au Lecteur de l'Interprect témoigne, à propos du basque, d'une conscience aiguë de la variation diastratique (dans l'espace) des langues, relevant que le français et l'espagnol disposent à cet égard de " correction authorisée ou omologuée », ce qui vise clairement le réseau des Inventeurs du bon usage étudié par Danielle Trudeau (Paris, Ed. de Minuit, 1991). Qu'en pensait-il à propos de l'occitan, dont le guide trilingue naturellement ne souffle mot?

33) L'excellente édition établie en orthographe classique, présentée et traduite par Robert Darrigrand, Orthez, Centre d'Etudes du Protestantisme Béarnais, 2002, 374 p. vient de faire l'objet d'un retirage en 2009 pour son inscription au concours du CAPES d'occitan, la rendant à nouveau disponible. Concernant le genre de l'églogue dialoguée, on rappellera que celui-ci jouit en Béarn d'une solide réputation, de Pèir de Garros à la Pastourade Gascoue de son frère cadet en passant par le poème trilingue de Du Bartas.

34) Quatre actes en vers. Dernière éd., en graphie classique, par Michel Grosclaude, Orthez, Per Noste, 2001, (coll. 'La Civada'). Le personnage du père reçoit successivement, en vue d'instruire son fils, les représentants de trois professions frottées d'université, jurisconsulte, médecin, apothicaire, naturellement francophones, qui l'horripilent, puis un maître d'armes que chassent les femmes de la maison : le fils sera paysan comme son père. La Nouvelle pastorale, qui suit, inverse la donne : les dix fils d'un patriarche archétypal, aux noms tous bibliques, se voient proposer autant de métiers déterminés dont ils dénoncent les travers moraux respectifs. A l'inverse du fils prodigue, ils resteront paysans. La revendication d'identité n'a cependant plus la placide solidité corporative des marchands et sent la manipulation physiocrate.

\section{APPENDICES}

1- [Nous pouvons proposer en appendice une édition moderne des deux poèmes gascons de Voltoire (62 vers) évoqués supra p. 13, seule production littéraire occitane conservée de Voltoire.]

2- $\quad$ IIl peut être joint, sous réserve d'autorisation de la Bibliothèque Municipale de Bordeaux, une reproduction de la table des matières de l'ouvrage perdu (commodément reproductible à partir du reprint procuré par X. Videgain, référencé supra, note 2).] 